\title{
Electrical and Acoustic Parameters of Wire-Guided Discharges in Water: Experimental Determination and Phenomenological Scaling
}

\author{
Y. Sun, I. V. Timoshkin, Senior Member, IEEE, S. J. MacGregor, Senior Member, IEEE, \\ M. P. Wilson, Member, IEEE, M. J. Given, Senior Member, IEEE, T. Wang, N. Bonifaci
}

\begin{abstract}
The present paper is focused on investigation of the electrical, hydrodynamic and acoustic parameters of underwater plasma discharges, stabilized with thin copper wires. The experimental current and acoustic waveforms have been obtained using different combinations of the circuit capacitance, charging voltage and wire length. The resistances of plasma discharges have been calculated for all combinations of electrical and topological parameters, based on the constant resistance approach. Phenomenological scaling relationships that link the plasma resistance and the total energy delivered to the plasma, the period of discharge cavity oscillation and the peak magnitude of the acoustic impulse have been obtained. These relationships can be used in optimization of the acoustic output from the wire-guided discharges for different practical applications.
\end{abstract}

Index Terms - Wire-guided underwater discharges, acoustic impulses, plasma resistance.

\section{INTRODUCTION}

$\mathrm{U}$ NDERWATER spark discharges generate strong acoustic and pressure impulses that can be used in different practical applications, for example in mineral engineering [1], rock drilling operations [2], material recycling [3, 4], and for environmental applications such as removal of pollutants in water [5]. The breakdown field in water depends on the duration of the high voltage (HV) impulses applied to the electrode system. For shorter HV impulses, with duration of a few tens of nanoseconds, the breakdown field could be as high as $\sim 1.5 \mathrm{MV} / \mathrm{cm}$; with an increase in the impulse duration, the breakdown field reduces to $\sim 0.1 \mathrm{MV} / \mathrm{cm}$ for $\mathrm{HV}$ impulses of microsecond duration [6]. Thus, for initiation of free spark discharges in water in centimeter-long gaps, significantly high applied voltages are required, which may lead to practical problems such as increased cost of dielectric insulation, increased overall size of the driving pulsed power systems and

This manuscript has been submitted for publication on 7 February 2017, in its final form on XX XXX 2017. I. Timoshkin is grateful for hospitality and support from G2E Laboratory, CNRS, France, which allowed for his research visits to G2E Laboratory in Grenoble in 2017.

Y. Sun, I. Timoshkin, M. Given, M. Wilson, S. MacGregor are with University of Strathclyde, Department of EEE, Glasgow, UK, G1 1XW, (e-mail: ying.sun@strath.ac.uk; igor.timoshkin@strath.ac.uk; m.given@strath.ac.uk;

scott.macgregor@strath.ac.uk).

N. Bonifaci is with the G2E Laboratory, CNRS, Grenoble, France. (e-mail: nelly.bonifaci@g2elab.grenoble-inp.fr) potential flashover problems. In the case of free discharges in water, there is limited control of the discharge path, and elevated conductivity of the water (for example, tap water or sea water) results in significant pre-breakdown energy losses due to Joule heating. Thus, the acoustic efficiency of free discharges in water is limited.

In order to minimise the pre-breakdown energy losses and to increase the acoustic efficiency of underwater spark discharges through the use of longer inter-electrode gaps, while maintaining lower levels of applied voltage, underwater spark discharges may be initiated with a thin conductive wire placed between the high voltage and ground electrodes.

A typical chain of events in the wire-guided discharge can be described as follows. The pulsed power circuit is closed by the thin conductor placed between the discharge electrodes, therefore significant conduction current starts to flow through the wire after application of the high voltage impulse. This current results in heating, melting and disintegration of the wire into fine droplets of the melted metal. After wire disintegration, the circuit becomes open, thus the conduction current collapses and the voltage across the entire inter-electrode gap increases until the eventual breakdown of the gas in this channel, which is filled with the droplets of melted metal. Upon breakdown, the energy stored in the pulsed power system is delivered into the plasma channel formed between the electrodes [7]. Typically, the energy required for wire heating, melting and disintegration constitutes only a small fraction of the total energy available in the discharge [8]. Therefore, the remaining energy is delivered into the breakdown channel formed by the disintegrated wire. The vaporised metallic wire defines the path of the post-breakdown plasma channel, which enhances the consistency and stability of such underwater discharges.

Wire-guided discharges in water can be utilised for different industrial applications and for research purposes. For example, fast electrical disintegration of metallic conductors in water can be used for fabrication of nanomaterials (metallic and metal oxide nanoparticles) [9], and for generation of high residual stresses in metallic parts [10]. Underwater wire-guided discharges are used in investigation of the conductivity and equations of state of warm dense matter [11,12]. Water has low compressibility, which results in slow radial expansion of the plasma developed in the initial stage of the underwater wire discharge, $\sim 10^{5} \mathrm{~cm} / \mathrm{s}$. This expansion velocity is almost two orders of magnitude lower than the plasma expansion velocity in the case of a wire explosion in vacuum [11]. Therefore, due to the high current magnitude and high plasma density 
developed in an underwater plasma channel, the equation of state of the warm dense matter formed in the breakdown channel, and the electrical conductivity of this matter, can be obtained and investigated [12].

Although wire-guided underwater discharges have been intensively studied for several decades, there is a lack of understanding of the relationships between their electrical, hydrodynamic and acoustic parameters. Thus, it is important to investigate the functional behavior of the electrical, hydrodynamic and acoustic parameters of the wire-guided discharges, in order to optimise and to tailor their acoustic output for practical applications. As in the case of free breakdown in water, underwater wire-guided discharges generate strong pressure impulses. The primary pressure impulse is produced at a very early stage of expansion of the plasma generated by the breakdown in the channel filled with gas and metallic droplets formed by the heated and disintegrated wire. Intensive Joule heating of the plasma in this channel results in fast expansion of the plasma/gas-filled cavity, which continues until its kinetic energy is completely converted into potential energy accumulated in the surrounding water. At this stage, the external hydrodynamic pressure is significantly higher than the pressure inside the cavity, which leads to its collapse and the radiation of a secondary pressure impulse. This dynamic process is similar to the expansion-collapse process of a cavity formed by a free discharge in water. The functional behavior of the period of cavity oscillation, the acoustic magnitude of the pressure impulses generated, and the resistance of the underwater plasma generated by free spark discharges in water have been investigated in a number of papers, including [13, 14].

Also from the late $1960 \mathrm{~s}$, different scaling relationships for spark discharges in water have been proposed, however there is still a lack of a general phenomenological model(s) that would help researchers and engineers who are working in this area to predict and to optimize the hydrodynamic parameters of transient underwater cavities and the acoustic impulses generated by these cavities. In [15], for example a scaling relation between the peak acoustic magnitude and the product of the voltage and peak current in free discharges in water was obtained graphically. However, no investigation of the potential influence of different inter-electrode lengths on the peak acoustic magnitude was carried out. In [16], several models, including a model based on the energy balance equation for the plasma discharge in water, were discussed. Some of the approaches proposed in [16] have been tested and confirmed to be valid for free discharges in water which take place in relatively short (tens of millimeters) inter-electrode gaps. However, no model is given in [16] that could provide scaling relationships between the electrical, hydrodynamic and acoustic parameters for longer discharges initiated with thin conductive wires. It is important to address this issue and to development of phenomenological scaling relationships for such discharges which will link their electrical and acoustic parameters and will enable their optimisation and tailoring.

The present paper is focused on an experimental investigation of such parameters for underwater wire-guided discharges with different wire/gap lengths as the charging voltage and capacitance of the pulsed power supply was varied.
The behavior of the following parameters has been analysed: the resistance of the plasma channel; the period of cavity oscillation; and the acoustic pulse magnitude (peak pressure). This has allowed phenomenological scaling relationships between the resistance of the plasma channel, the energy available in the discharge, the period of the cavity oscillation and the peak acoustic magnitude to be established. The obtained functional dependencies can be used for development and optimization of plasma-acoustic sources for specific practical applications.

\section{EXPERIMENTAL SET-UP}

The experimental system consists of a pulsed driving circuit connected to electrodes submerged in water, and diagnostic devices for monitoring the electrical and acoustic parameters. This system is similar to the pulsed power system developed and used in [14] for studying free discharges in water. Several combinations of different HV capacitors (Maxwell Laboratories Ltd, USA) were used to achieve total (measured) energy-storage capacitances of $155 \mathrm{nF}, 267 \mathrm{nF}$ and $533 \mathrm{nF}$ in the single-stage pulsed power system. The capacitance was charged to voltages of $25 \mathrm{kV}, 30 \mathrm{kV}$ and $35 \mathrm{kV}$ by a DC power supply (Glassman High Voltage, series EH).

The conductive wire used to generate spark discharges was copper $(99.9 \% \mathrm{Cu})$, with a diameter of $0.05 \mathrm{~mm}$ (Goodfellow Ltd). For each discharge, a length of copper wire was clamped by two stainless-steel crocodile clips, which were solidly mounted vertically opposite to each other on two stainless-steel rods, within a wire sample holder. The upper crocodile clip was connected to the output of the HV pulsed power system while the bottom crocodile clip was grounded. The electrode holder was placed in the middle of a large water tank with dimensions of $2 \mathrm{~m}$ (length) $\times 1 \mathrm{~m}$ (depth) $\times 1 \mathrm{~m}$ (width). The wire lengths (gap distances between the crocodile clips) were chosen as $15 \mathrm{~mm}, 45 \mathrm{~mm}$ and $75 \mathrm{~mm}$.

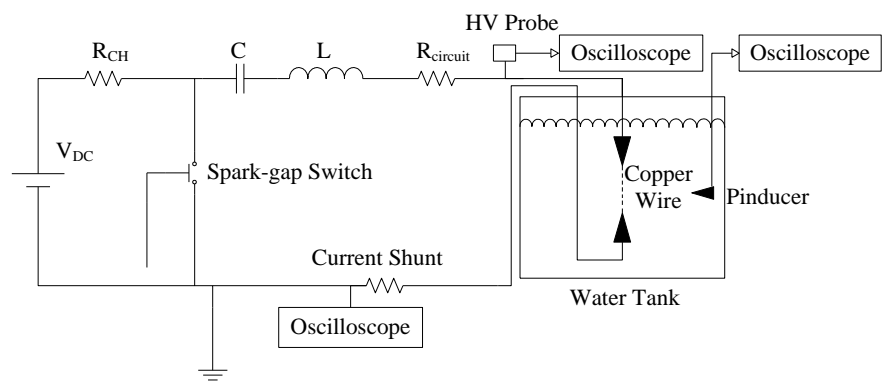

Fig.1. Schematic diagram of the experimental setup: $\mathrm{V}_{\mathrm{DC}}, \mathrm{DC}$ power supply; $\mathrm{R}_{\mathrm{CH}}$, charging resistor; $\mathrm{C}$, capacitor bank; $\mathrm{L}$, circuit inductance; $\mathrm{R}_{\text {circuit }}$, circuit resistance.

The diagnostic devices used in the present study are also as described in [14]. A high voltage probe (NorthStar PVM-5, 1000:1 division ratio, $80 \mathrm{MHz}$ bandwidth) and a resistive current shunt probe (Samtech Ltd DE(CP)-01, $29.6 \mathrm{~m} \Omega$ ) were used to capture the transient voltage and current waveforms, and the corresponding output signals were recorded by a Tektronix 2024 digitising oscilloscope with 2 GSample/s sampling rate and $200 \mathrm{MHz}$ bandwidth. The acoustic signals were registered by a piezoelectric acoustic sensor, Valpey 
Fisher Pinducer VP1093, and the output voltage signal from the Pinducer was transferred to a Tektronix 3054B digitising oscilloscope with $5 \mathrm{GSample} / \mathrm{s}$ sampling rate and $500 \mathrm{MHz}$ bandwidth. The acoustic sensor was located $500 \mathrm{~mm}$ away from the wire-guided discharge source. A schematic diagram of the experimental setup is given in Fig. 1.

\section{ELECTRICAL AND ACOUSTIC WAVEFORMS}

As described in Section II, the wire-guided spark discharges were initiated by a thin copper wire located between the HV and ground electrodes. Each combination of charging voltage $(25 \mathrm{kV}, 30 \mathrm{kV}$ and $35 \mathrm{kV}$ ) and capacitance (155 nF, $266 \mathrm{nF}$ and $533 \mathrm{nF}$ ) was used to generate discharges in tap water, stabilized by $\mathrm{Cu}$ wires with three different lengths: $15 \mathrm{~mm} ; 45 \mathrm{~mm}$ and $75 \mathrm{~mm}$. The transient voltage and current waveforms and corresponding acoustic output were recorded for each wire-guided discharge. Figure 2 shows an example of the discharge current waveform obtained with a $45 \mathrm{~mm}$-long wire.

The initial sharp current spike in Fig. 2 indicates the process of copper wire melting and disintegration. Upon applying the high voltage impulse to the wire, the current through the wire placed between the electrodes rises over a few tens of nanoseconds (initial current spike, dotted line in Fig.2). Due to the injected electrical energy, the wire is heated, melted and disintegrated. The resistance between the electrodes increases and the current drops.

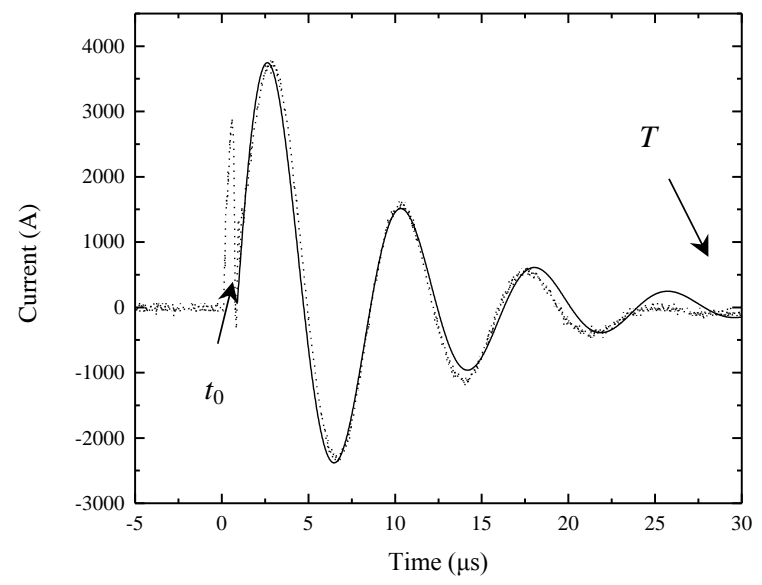

Fig.2. Current waveforms for wire-guided underwater spark discharge with the following parameters: $45 \mathrm{~mm}$-long wire, $266 \mathrm{nF}$ energy-storage capacitance, $17.5 \mathrm{kV}$ peak impulse voltage. The dotted line is the experimental current waveform $I(t)$; the solid line is the analytical fitting by (1). Analytical fitting starts at time $t_{0}$ - the moment of the breakdown in the channel formed by the disintegrated wire. $T$ indicates the end of the energy deposition phase.

Due to the high resistance now present across the gap, the inter-electrode voltage increases, causing breakdowns of a gaseous nature between the droplets of melted copper in the channel formed by the disintegrated wire, reclosing the inter-electrode gap. The current oscillations in the pulsed discharge circuit can now be described by under-damped oscillations in a $R-L-C$ circuit (starting from time $t_{0}$ in Fig.2). By simplifying the discharge circuit to a lumped $R-L-C$ circuit, and assuming that the resistance of the breakdown plasma channel is constant, the current oscillations after wire disintegration and the final breakdown of the channel formed by the disintegrated wire can be fitted with the function:

$$
I(t)=I_{0} \exp \left(-\frac{R_{\text {total }}}{2 L} t\right) \sin \left(t \sqrt{\frac{1}{L C}-\left(\frac{R_{\text {total }}}{2 L}\right)^{2}}\right)
$$

where $I_{0}$ is a fitting constant related to the maximum peak current in the transient process, and $R_{\text {total }}$ is the total resistance of the experimental circuit, including the assumed constant resistance of the plasma channel, $R_{p l}$.

In Fig.2, the oscillations shown by the solid line indicates the current waveform obtained using (1), from the moment of the secondary breakdown in the channel, $t_{0}$, until the end of the energy deposition stage, $T$. The dotted line shows the obtained experimental current waveform. The match between the experimental waveform and the current waveform plotted using (1) provides validation of the use of the constant resistance approach in the analysis of the underwater discharges.

Using (1), the circuit inductance, $L$, and the total resistance, $R_{\text {total }}$, were obtained. The constant resistance of the plasma channel, $R_{p l}$, can be calculated by subtracting the derived circuit resistance, $R_{\text {circuit }}$, obtained from current waveforms, using (1), when the electrode gap was short circuited (no contribution from the spark discharge channel), from the total resistance, $R_{\text {total }}$, of the circuit with the spark discharge channel, also obtained using (1):

$$
R_{\text {pl }}=R_{\text {total }}-R_{\text {circuit }}
$$

The acoustic waveforms emitted by the wire-guided discharges were monitored and recorded. The primary acoustic pressure impulse emitted by the expanding plasma cavity was registered by the Tektronix digitizing oscilloscope using a time base of $200 \mathrm{~ns} /$ division, while the acoustic signals generated by the first complete expansion-collapse cycle of the cavity were captured using a longer time base of $1 \mathrm{~ms} /$ division.
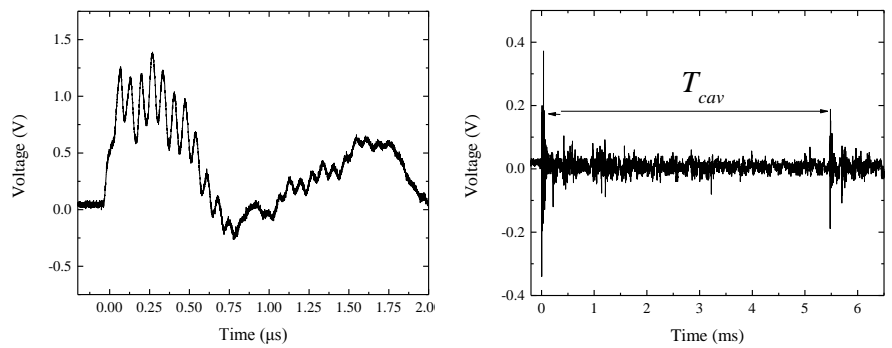

Fig.3. Acoustic signals generated by the underwater wire-guided discharge. (a), primary signal due to cavity expansion; (b) primary and secondary signals generated by expanding and collapsing cavity $(15 \mathrm{~mm}$ length wire, $20.8 \mathrm{kV}$ impulse voltage, $155 \mathrm{nF}$ energy-storage capacitance). $T_{\text {cav }}$ is the time interval between the primary pressure impulse due to cavity formation and the secondary pressure impulse due to cavity collapse (the period of cavity oscillation)

Fig.3 shows a typical example of the acoustic output registered from a 15-mm-long wire-guided discharge, with the 
acoustic waveforms of the primary acoustic impulse generated by the formation of the cavity (Fig. 3(a), short timescale) and the primary and secondary acoustic impulses radiated by the formation and collapse of the cavity (Fig. 3(b), longer timescale). The magnitude of the primary acoustic impulses, $P_{a c}$, is measured as the direct output signal from the acoustic sensor in Volts, following the procedure established in [14]. In Fig. 3(b), the period of the cavity oscillation, $T_{\text {cav }}$, is read as the time interval between the formation and collapse of the cavity. These two parameters, $P_{a c}$ and $T_{c a v}$, are used in further investigation of the performance of the wire-guided discharges.

\section{ELECTRICAL, HYDRODYNAMIC AND ACOUSTIC PARAMETERS OF WIRE-GUIDED DISCHARGES}

The resistance of the plasma in the breakdown channel is a key factor defining the acoustic and hydrodynamic performance of the plasma channel. The relationship between the plasma resistance, $R_{p l}$, and the circuit resistance, $R_{\text {circuit }}$, determines the energy partition in the circuit and, thereby, the amount of electrical energy transformed into acoustic energy. In general, the plasma resistance of a channel is related to the length of the channel. The path of the wire-guided plasma channel is defined (stabilized) by the wire, and therefore follows the shortest distance between the electrodes. Thus, because of this gap stabilization, the plasma resistance is consistent for breakdown events under the same energisation conditions, and with the same gap length. Therefore, the correlation between the plasma resistance and other discharge parameters, such as energy available in the discharge, can be established and used in the analysis of the hydrodynamic and acoustic properties of wire-guided spark discharges. In this experimental study, it was found that the plasma resistance across all combinations of wire length, voltage and capacitance varied from $0.56 \Omega$ to $2.29 \Omega$.

\section{A. Electrical parameters}

In the case of spark breakdown in fluids, the transient resistance of the breakdown channel demonstrates a highly dynamic behavior, and typically decreases during a few tens/hundreds of nanoseconds from its initial, relatively high, value, to a minimum value of a few tens/hundreds of milliohms $[17,18]$. There are several models that describe this dynamic resistance of the spark breakdown channel as a function of time. For example, based upon the plasma energy balance equation in the breakdown channel, it has been shown that the transient resistance of the breakdown channel in a gas, $R(t)$, is inversely proportional to the integral of $I(t)^{2}$, and directly proportional to the length of the breakdown channel, $\ell$ :

$$
R(t)=k \frac{\ell}{\left(\int_{t_{0}}^{t} I(t)^{2} d t\right)^{n}}
$$

where $n$ is a constant. According to the energy balance model developed by Rompe and Weizel (and discussed in [18]), $n$ in
(3) is equal to $1 / 2$ and coefficient $k$ (noted as $k_{R W}$ for the Rompe - Weizel's model) is a function of different parameters of the plasma breakdown channel including electronic temperature, electronic mobility, and ionization potential. $k_{R W}$ is typically considered as a constant for a specific range of the experimental parameters, [18]. In Vlastos's energy balance model, $n=3 / 5$ [19] and coefficient $k$ (noted as $k_{W}$ in this model) is considered as a model constant which also depends on the plasma parameters and the initial radius of the plasma channel, [19]. The detailed analysis of coefficients $k_{R W}$ and $k_{W}$ is beyond the scope of this work, further details related to these coefficients and their numerical values for transient spark discharges in gas including discharges initiated with thin conductive conductors can be found in $[18,19,20]$. Popovic et al. generalised this approach in [21], by assuming that $n$ is a phenomenological constant that can be obtained using the best fit between the calculated and experimental values of the breakdown channel resistances. It was shown in $[21,22]$ that using (3) with $n=1 / 2$ and $n=3 / 5$ provides reasonable agreement with the experimentally-obtained transient spark resistance in air. Moreover, it was found in [20] that (3) with $n=1 / 2$ can be used for description of the transient resistance of the breakdown channel in liquids: Fuhr and Schmidt used (3) in their analysis of the transient breakdown resistance in liquid hydrocarbons. In the present paper, (3) is used to provide a link between the electrical and hydrodynamic parameters of the underwater wire-guided discharges. The phenomenological coefficient, $n$, was obtained for all combinations of the circuit capacitance, charging voltage and wire length, using the fitting procedure described later in this section.

After fast post-breakdown reduction, the plasma resistance remains almost constant until the end of the energy deposition process, which can last for a few tens of microseconds [18, 20]. Thus, in the case of the transient processes of such duration or longer, it is possible to substitute the transient resistance with an effective (constant) spark plasma resistance. For example, a constant plasma resistance was used to describe the transient current oscillations observed for short, sub-micrometer and millimeter air gaps [23], and for long, hundreds of centimeters, air gaps [24]. As shown in [20], the calculated resistance at the end of the transient process obtained using (3) is very close to the experimentally-obtained resistance of the breakdown channel. Therefore, it can be postulated that the efficient (constant) plasma resistance, $R_{p l}$, is proportional to the transient resistance, $R(T)$, at the end of current oscillation obtained using (3) at $t \rightarrow T$.

In the present work, it is assumed that the transient resistance of the breakdown channel at the end of the energy deposition phase is proportional to the constant resistance value of the plasma channel obtained by the fitting procedure based on (1):

$$
R(T) \propto R_{p l}
$$

To provide a link between the acoustic and hydrodynamic parameters of the underwater wire-guided discharges, and the total energy deposited into the plasma channel, $W(T)$, the total energy has been calculated by integrating the current waveforms, using the values of $R_{p l}$ obtained from (1) and (2): 


$$
W(T)=R_{p l} \int_{t_{o}}^{T} I(t)^{2} d t
$$

where the integration is conducted from $t_{0}$, the moment of the breakdown in the gas/liquid metal droplets channel formed by the disintegrated wire, until the end of the energy deposition phase, $T$, Fig.2. The total energy deposited into the plasma channel, $W(T)$, has been obtained for all combinations of the charging voltage, capacitance and wire length. The validity of the constant resistance approach to calculation of the energy deposited into the underwater breakdown channel has been discussed and justified in [13].

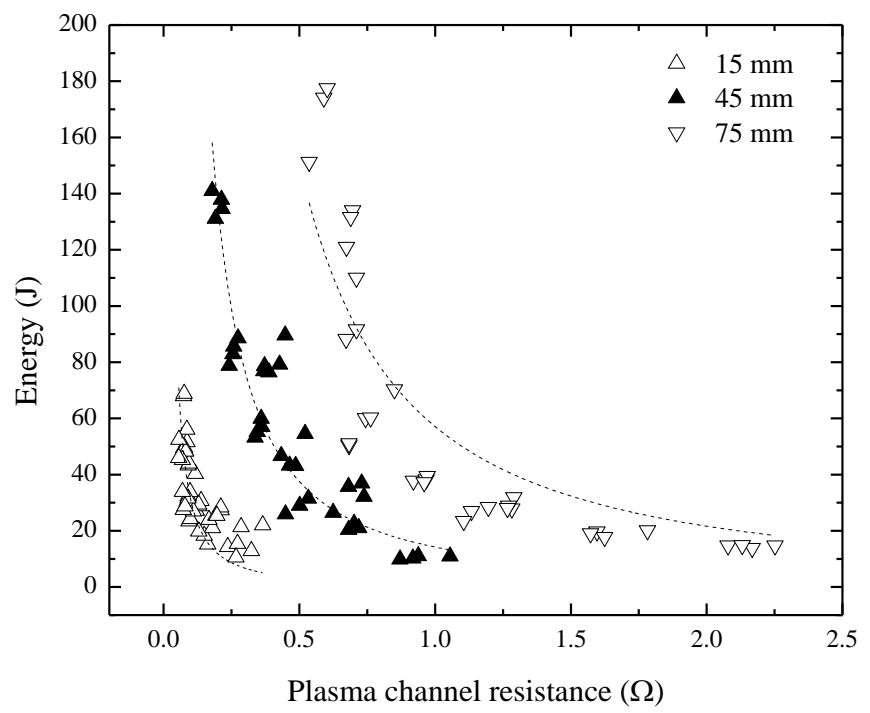

Fig.4. Plasma energy as a function of constant plasma resistance for all three capacitances and three gap distances. Dashed lines, analytical fitting using (6), $n=0.41 \pm 0.03$.

Thus, one can obtain the link between the energy deposited into the plasma channel, $W(T)$, and the resistance of the plasma channel, $R_{p l}$, by combining (3), (4) and (5):

$$
W(T)=a\left(\frac{\ell}{R_{p l}^{1-n}}\right)^{\frac{1}{n}}
$$

The total energy, $W(T)$, obtained using the experimental current waveforms was fitted with analytical current functions given by (6). Coefficient $a$ in (6) is a phenomenological constant. The calculated values of $W(T)$ and the corresponding fitting lines are shown in Fig. 4 as functions of the plasma resistance, $R_{p l}$, for all wire lengths of wires and for all tested combinations of charging voltage and capacitance. The dashed lines in Fig. 4 represent analytical fittings of (6), obtained using Origin 8 graphing software with $n=0.41 \pm 0.03$, and $a=(14.33 \pm 0.12) \times 10^{-3}$. Thus, this analytical fitting procedure demonstrates that the phenomenological relationships (4) and (6) can be used for satisfactory scaling of underwater wire-guided discharges.

\section{B. Hydrodynamic parameters}

The phenomenological scaling relationship between the period of cavity oscillation, $T_{\text {cav }}$, and the energy available in the discharge, $W$, has been tested in [14] for free discharges in water, and found to be valid for different combinations of electrical (breakdown voltage, energy-storage capacitance) and topological (inter-electrode distance) parameters:

$$
T_{c a v} \propto W^{\frac{1}{3}}
$$

Coefficient proportionality between $T_{c a v}$ and $W$ in (7) is a model coefficient which is a function of hydrodynamic and energy parameters including a portion of the total electrical energy released in the breakdown plasma channel which is converted into thermal energy in the plasma, hydrostatic pressure and specific weight of liquid (water), [14].

Assuming the same functional dependency between the period of oscillations and the delivered energy, $W(T)$, for wire-guided discharges in water, the combination of (6) and (7) allows for establishment of the relationship between the period of cavity oscillations and the plasma resistance:

$$
T_{c a v}=b\left(\frac{\ell}{R_{p l}^{1-n}}\right)^{\frac{1}{3 n}}
$$

where $b$ is a phenomenological coefficient which is a function of the proportionality coefficients in (6) and (7).

The period of cavity oscillations, $T_{c a v}$, has been obtained for all combinations of $\ell, \mathrm{C}$ and charging voltage.

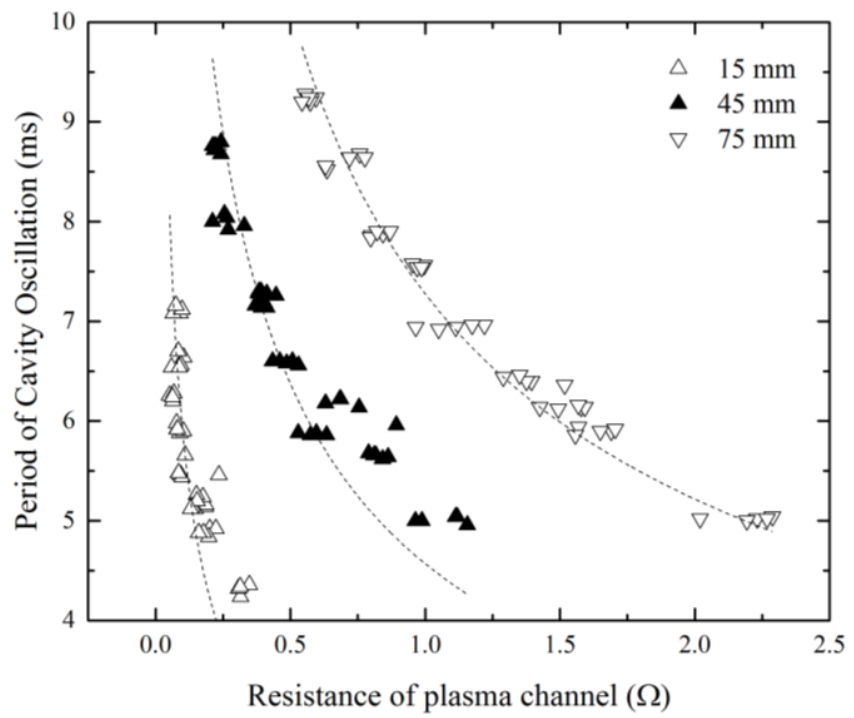

Fig.5. Period of cavity oscillation as a function of the plasma resistance for all three capacitances: $155 \mathrm{nF}, 266 \mathrm{nF}$ and $533 \mathrm{nF}$; and all three wire lengths: $15 \mathrm{~mm}, 45 \mathrm{~mm}$, and $75 \mathrm{~mm}$. Dashed lines represent analytical fitting lines according to (8), with $n=0.41 \pm 0.04$.

Fig.5 presents the obtained period of cavity oscillation, $T_{c a v}$, as a function of the plasma resistance, $R_{p l}$. It was found that the 
period of cavity oscillation decreases as the plasma resistance increases for each specific length of wire, and the period of cavity oscillation is longer for either higher capacitance or longer wire length (longer inter-electrode gap). The dashed lines in Fig.5 represent the analytical fitting lines obtained using (8). The mean values of coefficients $n$ and $b$ in (8) that provides the best fit to all experimental data for all three wire lengths are $n=0.41 \pm 0.04$ and $b=0.22 \pm 0.01$. Thus, the close agreement in the value of $n$ obtained for the fit of (6) to the data for energy deposition, and for the fit of (8) to the data for the oscillation period of the cavity, confirms the validity of the approach used to describe the electrical and hydrodynamic parameters of wire-guided discharges in water.

\section{Acoustic parameters}

The peak acoustic magnitude of the pressure impulses generated by the wire-guided discharges as a function of the plasma resistance was obtained for all combinations of capacitance, wire length and charging voltage. Fig. 6 shows the peak acoustic magnitude as a function of the plasma channel resistance - it was found that, for each specific wire length, the peak acoustic magnitude decreased with an increase in $R_{p l}$.

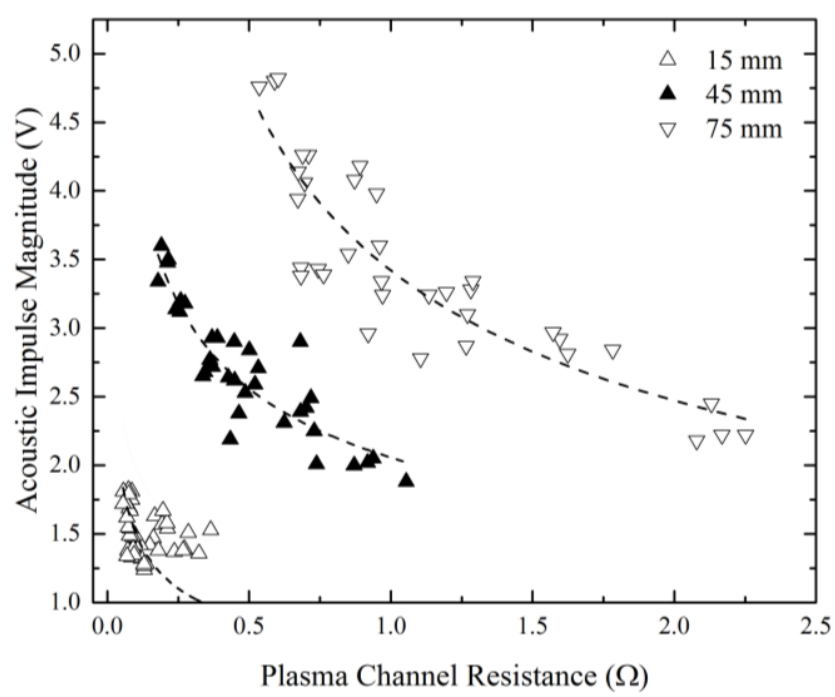

Fig.6. Acoustic emission magnitudes as a function of the plasma resistance for all three capacitances: $155 \mathrm{nF}, 266 \mathrm{nF}$ and $533 \mathrm{nF}$; and all three wire lengths. Dashed lines represent analytical fitting lines according to (9).

Fig.6 shows that, for the same plasma resistance, the peak magnitude of the acoustic signal generated with longer wires is larger than the acoustic magnitude generated by the discharges stabilized with shorter wires. However, for each wire length, the peak acoustic magnitude, $P_{a c}$, generated using all three tested capacitances and charging voltages, demonstrates similar functional dependency on the plasma resistance, $R_{p l}$, and the length of wire, $\ell$ : the peak acoustic magnitude is proportional to the length of wire, and inversely proportional to the plasma resistance to the power $m$ :

$$
P_{a c}=c_{1} \frac{\ell}{R_{p l}^{m}}
$$

where $c_{l}$ and $m$ are phenomenological constants that can be obtained by fitting (9) to the experimental data shown in Fig.6. Again using Origin Pro 8, the value of coefficients $m$ and $c_{1}$ that provided the fit for all three wire lengths were $m=0.37 \pm 0.07$ and $c_{1}=(4.43 \pm 0.15) \times 10^{-2}$.

Thus, it is shown that in the case of stabilization of the discharge channel with a thin wire, the acoustic magnitude generated by the discharges is proportional to the length of the wire. This is in agreement with [25], where an analytical equation for the total acoustic energy in the pressure impulse generated by an underwater discharge was shown to be directly proportional to the integral of the squared pressure signal and the length of the wire, $\ell$. Using (6) and (9), the link between the peak acoustic magnitude, $P_{a c}$, and the total energy deposited into the plasma channel, $W(T)$, can be derived:

$$
P_{a c}=c_{2}\left(\ell^{(1-n-m)} W(T)^{n m}\right) \frac{1}{1-n}
$$

where coefficient $c_{2}$ is a function of coefficients $c_{1}$ and $a$, $c_{2}=c_{1} a^{n m /(n-1)}$. Inserting the obtained numerical values for the coefficients, $n=0.41$, and $m=0.37$, (10) can be represented as:

$$
P_{a c}=c_{2} \ell^{0.37} W(T)^{0.26}
$$

Equation (11) follows the form of the empirical relationship between the peak acoustic magnitude produced by a spark discharge in water and the corresponding energy available in the spark discharge used in [26], where it was shown that $P_{a c} \propto W^{\beta / 3}$, with $\beta=1.13$. It can be seen that the power coefficient, $\beta / 3$, used in [26] is close to the power coefficient, 0.26 , obtained in the present work.

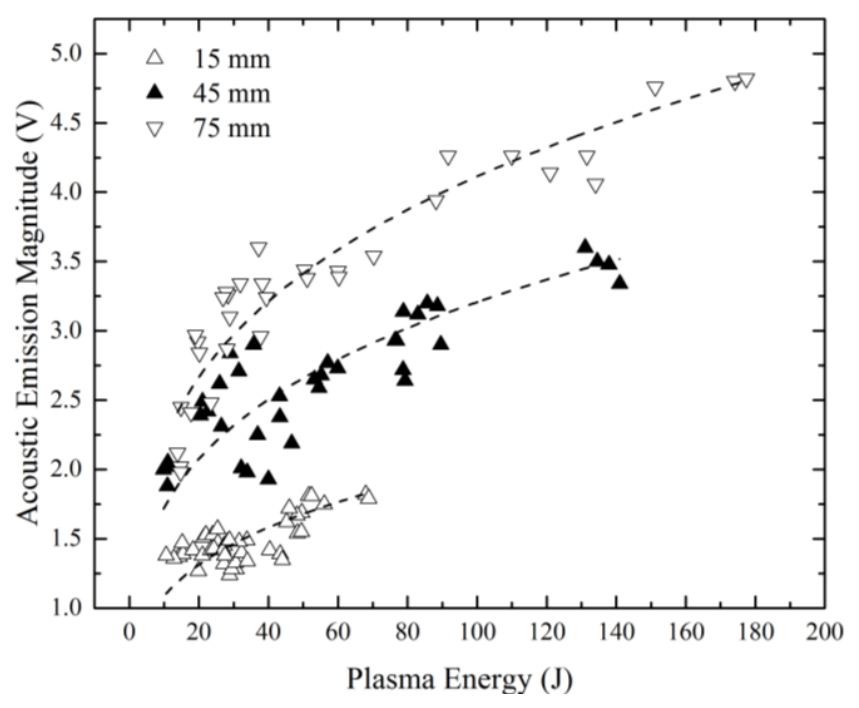

Fig.7. Acoustic emission magnitudes as a function of the energy available in discharges for all three capacitances: $155 \mathrm{nF}, 266 \mathrm{nF}$ and $533 \mathrm{nF}$; and for all three wire lengths: $15 \mathrm{~mm}, 45 \mathrm{~mm}$, and $75 \mathrm{~mm}$. Dashed lines represent analytical fitting lines according to (11).

Fig.7 shows experimental values of the peak acoustic magnitude plotted against the total energy available in the 
wire-guided discharge for all three wire lengths, all capacitances and all charging voltages. The dashed lines represent the fitting obtained by (11), $c_{2}$ was a variable parameter, $c_{2}=0.22 \pm 0.02$, which confirms the value of $c_{2}=0.238$ obtained using combination of (6) and (9). The good fit between the experimental data and the behavior predicted by (11) confirms that the phenomenological scaling relationships proposed in the present work can satisfactorily describe the functional dependency of the acoustic magnitude on the energy delivered to the discharge and the plasma resistance. These relationships provide a mechanism for the optimization of practical acoustic wire-guided discharge sources.

\section{CONCLUSIONS}

The main objectives of the present paper include experimental determination of the electrical and acoustic parameters of the underwater wire-guided discharges, and the development of phenomenological scaling relationships for these discharges. A detailed experimental investigation of the behavior of wire-guided underwater discharges has been made over a range of wire/gap lengths, charging voltages and energy-storage capacitances. The electrical parameters: plasma resistance (assumed constant) and the energy delivered into the plasma channel, together with the period of cavity oscillation, a hydrodynamic parameter, and the magnitude of the acoustic impulses have been either measured or derived. The relationships between the total energy delivered into the plasma channel, the period of cavity oscillation, the acoustic magnitude and the plasma resistance have been investigated and fitted with phenomenological equations, based on the dependency of the plasma resistance on the integral of the current delivered to the plasma channel.

The present paper also is focused into the development of scaling relationships for wire-guided underwater discharges. It was shown that, based on basic relationships between the plasma resistance and its relationship with the current flowing in the discharge channel, it is possible to derive phenomenological scaling equations for wire-guided underwater discharges. These phenomenological equations have been shown to describe satisfactorily the main electrical, hydrodynamic and acoustic parameters of wire-guided discharges. The relationships include the wire/gap length as a parameter, together with the total energy delivered into the discharge, and the plasma resistance. Thus, the sensitivity of the discharge parameters to the length of wire has been captured in the proposed equations. The established scaling relationships have a different character than the corresponding relationships for free discharges in water [14]: the role of the inter-electrode gap in the free discharges is different, and it was shown that the main hydrodynamic and acoustic parameters of these discharges can be described by relationships which include the circuit parameters only as free variables. However, as has been shown in the present work, for longer inter-electrode gaps stabilized with conductive wires, the inter-electrode gap (the wire length) plays an independent role and must be included in the scaling relationships as a free parameter.

The obtained experimental results and the phenomenological scaling relationships will help in further optimization and development of practical applications of underwater wire-guided discharges.

\section{REFERENCES}

[1] T. Parker, F. Shi, C. Evans, M. Powell, "The effects of electrical comminution on the mineral liberation and surface chemistry of a porphyry copper ore", Minerals Engineering, vol. 82, no.15 2015, pp. 101-106.

[2] I. Timoshkin, J. Mackersie, S. MacGregor, "Plasma channel miniature hole drilling technology", IEEE Transactions on Plasma Science, vol.32, no.5, pp. 2055-2061.

[3] H. Bluhm, W. Fre , H. Giese, P. Hoppe, C. Schultheis , R. Strassner, "Application of pulsed $\mathrm{HV}$ discharges to material fragmentation and recycling", IEEE Trans. Dielectr. Electr. Insul. vol.7, no.5, pp. 625-636, 2000.

[4] M. Wilson, L. Balmer, M. Given, S. MacGregor, I. Timoshkin, "An investigation of spark discharge parameters for material processing with high power ultrasound", Minerals Engineering, vol.20, n.12, pp. 1159-1169

[5] T. Dang, A. Denat, O. Lesaint, G. Teissedre, "Pulsed electrical discharges in water for removal of organic pollutants: a comparative study", The European Physical Journal - Applied Physics, vol.47, no.2 2009, 22818

[6] M. Hogg, I. Timoshkin, M. Given, M. Wilson, S. MacGregor, T. Wang, R. Fouracre, J. Lehr, "Impulse breakdown of water with different conductivities," IEEE Transactions on Dielectrics and Electrical Insulation, vol. 19, no. 5, pp. 1559-1568, 2012.

[7] "Exploding Wires", vol.2, Ed. by W. Chase and H. Moore, Plenum Press, NY, 1962

[8] L. Nelson, S. Hogelandb, T. Roth, "Aluminum-Enhanced Underwater Electrical Discharges For Steam Explosion Triggering", Sandia National Laboratories, Report $\quad$ SAND99-0796, 1999, https://www.osti.gov/scitech/servlets/purl/12653

[9] C. Peng, J. Wang, N. Zhou, G. Sun, "Fabrication of nano-powders by electrical explosion of a copper wire in water", Current Appl. Phys., vol. 16, pp.284-287, 2016

[10] I. Timoshkin, S. MacGregor, Rolls-Royce PLC (Patent applicant), "Apparatus and method for electric spark peening of gas turbine components", Patent No. 20100008786, 2010.

[11] Y. Krasik, S. Efimov, D. Sheftman, et al, "Underwater electrical explosion of wires and wire arrays and generation of converging shock waves", IEEE Transactions on Plasma Science, vol.44, no.4, pp. 412-431, 2016

[12] T. Sasaki, Y. Yano, M. Nakajima, T. Kawamura, "Warm-dense-matter studies using pulse-powered wire discharges in water", Laser and Particles Beams, vol. 24, no. 3, 2006, pp. 371-380

[13] I. Timoshkin, R. Fouracre, M. Given, S. MacGregor, "Hydrodynamic parameters of the transient underwater cavities" J. Phys. D: Appl. Phys. vol.39, n.22, pp.4808, 2006.

[14] Y. Sun, I. Timoshkin, M. Given, M. Wilson, T. Wang, S. MacGregor, N. Bonifaci, "Impulsive Discharges in Water: Acoustic and Hydrodynamic Parameters", IEEE Transactions on Plasma Science, vol. 44, no. 10, pp. 2156-2166, 2016.

[15] W. Guman, B. Humphrey Jr., "Studies on an electric discharge underwater sound source", Report No. FHR-2229-7, Fairchild Hiller Corp Farmingdale NY Republic Aviation Div, 1968, http://www.dtic.mil/cgi-bin/GetTRDoc?AD=AD0681862

[16] K. Naugol'nykh and N. Roi, "Spark discharges in water (A hydrodynamical description)", (Translation, Foreign Technology Division), Wright-Patterson AFB, Dayton, OH, USA, Tech. Rep., 1974.

[17] T. G. Engel, A. L. Donaldson, and M. Kristiansen, "The Pulsed Discharge Arc Resistance and its Functional Behavior," IEEE Transactions on Plasma Science, vol. 17, no. 2, pp. 323-329, 1989

[18] R. Montano, M. Becerra, V. Cooray, M. Rahman, P. Liyanage, "Resistance of Spark Channels," IEEE Transactions on Plasma Science, vol. 34, no. 5, pp. 1610-1619, 2006

[19] A. Vlastos, "The Resistance of Sparks," J. Appl. Phys., vol. 43, no. 4, pp. 1987-1989, 1972

[20] P. Castera, P.-Q. Elias, "Resistance models applied to the return stroke phase of negative pulsed surface discharge in air", IEEE Transactions on Plasma Science, vol. 42, no. pp. 1922-1931, 2014 
[21] M. M. Popovic, S. S. Popovic, M. M. Platisa, "Investigation of the beginning of high current discharges in pulsed arcs," in Proc. Of Third Int. Conf. on Gas Discharges, The Institution of Electrical Engineers (IEE), Publication no.118, London, 1974, pp. 32-36.

[22] J. Fuhr, W. Schmidt, "Spark breakdown of liquid hydrocarbons. II. Temporal development of the electric spark resistance in n-pentane, n-hexane, 2,2 dimethylbutane, and n-decane", J. Appl. Phys., vol. 59, no. 11, pp. 3702-3708, 1986

[23] W. D. Greason, "Methodology to study the resistance of spark discharges", IEEE Trans. Ind. Appl., vol. 35, no. 2, pp. 359-365, 1999

[24] F. Théberge, J.-F. Daigle, J.-C. Kieffer, F. Vidal, M. Châteauneuf, "Laser-guided energetic discharges over large air gaps by electric-field enhanced plasma filaments", Sci. Rep. vol.7, 40063; doi: 10.1038/srep40063, 2017

[25] A. Grinenko, V. Gurovich, Ya. Krasik, A. Sayapin, S. Efimov, J. Felsteiner, "Analysis of shock wave measurements in water by a piezoelectric pressure probe", Review Of Scientific Instruments, vol.75, no.1, 2004, pp.240-244.

[26] J. McGrath, "Scaling Underwater Exploding Wires", NRL Report 6266, Energy Conversion Branch, Electronic Division, US Naval Research Laboratory, Washington, 1965

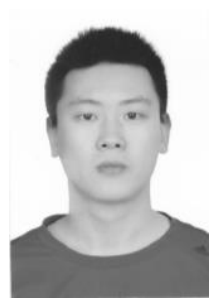

Ying Sun was born in Shandong, China. He received the B.Eng degree in electronic and electrical engineering in 2012 and M.Sc degree in electrical power engineering with business in 2013 from University of Strathclyde, Scotland, United Kingdom. He is currently pursuing the Ph.D degree in high voltage technology at University of Strathclyde,

Scotland, UK.

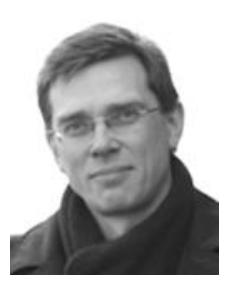

Igor V. Timoshkin (M'07-SM'14) received a degree in physics from the Moscow State University (Russia) in 1992, and the Diploma and the Ph.D. degree from the Imperial College of Science, Technology and Medicine, (London, UK) in 2001. After graduation from MSU he worked as a Researcher at Moscow State Agro-Engineering University, and then at the Institute for High Temperatures of Russian Academy of Sciences before moving to ICSTM in 1997. He joined the Department of Electronic and Electrical Engineering of the University of Strathclyde (Glasgow, UK) in 2001 where he became a Reader in 2016. His research interests include transient spark discharges in gases and liquids, environmental applications of non-thermal plasma discharges, plasma air-cleaning and bio-decontamination technologies.

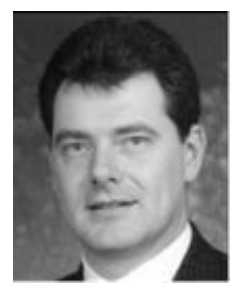

Scott J. MacGregor (M'95-SM'14) received the B.Sc. and Ph.D. degrees from the University of Strathclyde, Glasgow, U.K., in 1982 and 1986, respectively. He became a Pulsed Power Research Fellow in 1986 and a Lecturer in pulsed-power technology in 1989. In 1994, he became a Senior Lecturer, with a promotion to Reader and Professor of High Voltage Engineering, in 1999 and 2001, respectively. In 2006 and 2010 he became Head of the Department of Electronic and Electrical Engineering and Executive Dean of the Faculty of Engineering, and has been the Vice-Principal of the University of Strathclyde since 2014. Professor MacGregor was the recipient of the 2013
IEEE Peter Haas Award, and he was appointed as an Associated Editor of the IEEE Transitions on Dielectrics and Electrical Insulation in 2015. His research interests include high-voltage pulse generation, high-frequency diagnostics, high-power repetitive switching, high-speed switching, electronic methods for food pasteurization and sterilization, generation of high-power ultrasound (HPU), plasma channel drilling, pulsed-plasma cleaning of pipes, and stimulation of oil wells with HPU.

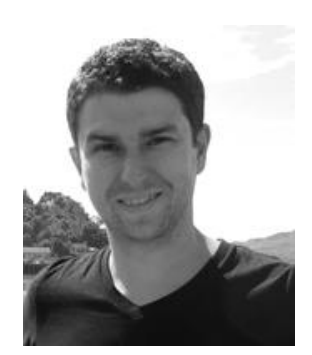

Mark P. Wilson (M'10) was born in Stranraer, Scotland, in 1982. He received the B.Eng. (with honours), M.Phil., and $\mathrm{Ph} . \mathrm{D}$. degrees in electronic and electrical engineering from the University of Strathclyde, Glasgow, U.K., in 2004, 2007, and 2011, respectively. $\mathrm{He}$ is presently based in the High Voltage Technologies research group at the University of Strathclyde, where his research interests include interfacial surface flashover, nanodielectrics, and the practical applications of high power ultrasound, corona discharges, and pulsed electric fields. Mark is a member of the IEEE Nuclear and Plasma Sciences Society, from whom he received a Graduate Scholarship Award in 2011, the IEEE Dielectrics and Electrical Insulation Society, and the IET.

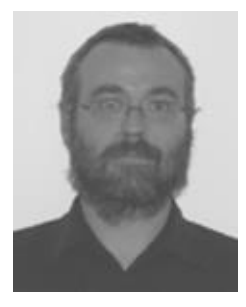

Martin J. Given (M'99-SM'11) is currently a Senior Lecturer in the Department of Electronic and Electrical Engineering at the University of Strathclyde. He received a degree in physics from the University of Sussex in 1981 and a $\mathrm{PhD}$ in electronic and electrical engineering from the University of Strathclyde in 1996. His research interests include, ageing processes and condition monitoring in solid and liquid insulation systems, high speed switching and pulse power.

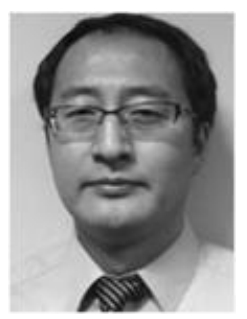

Tao Wang received the B.Eng and M.Sc degrees from Northeast China Dianli University (China) in 1993 and 1996 respectively, and the Ph.D. degree from the University of Strathclyde (Glasgow, UK) in 2005. He then joined the Newland Entech as a research fellow developing high efficiency industrial ozone generator. He joined the department of Electronic and Electrical Engineering of University of Strathclyde as a lecturer in 2010. His research interests include non-thermal gas discharges and their applications in gas synthesis, water disinfection and advanced oxidation process in water.

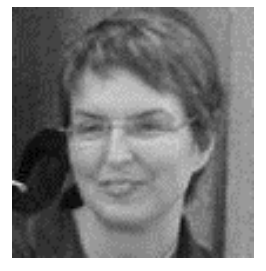

Nelly Bonifaci graduated from University Joseph Fourier with a MEng degree in Physics in 1989. In 2008 she obtained the HDR Habilitation qualification from the University of $\mathrm{J}$ Fourier, Grenoble. Dr Bonifaci joined the Laboratory on Dielectric Materials and Electrostatics 
(now G2E laboratory) CNRS, France in 1989 where she became a $1^{\text {st }}$ class researcher in 1997. In 2007 she was promoted to a position of a Senior Researcher/Team Leader. Dr Bonifaci research interests include breakdown in insulating liquids, plasma streamers in liquid dielectrics, pre-breakdown and plasma phenomena in dielectric liquids including corona discharges and electronic transport. Dr Bonifaci is an Editor of the IEEE Transactions of Dielectric and Electrical Insulation and a member of International Organising Committee of the IEEE Conference on Dielectric Liquids. 